This article may be downloaded for personal use only. Any other use requires prior permission of the author and AIP Publishing.

This article appeared in Surface Science Spectra 27, 014021 (2020) and may be found at https://avs.scitation.org/doi/10.1116/6.0000162. 


\section{Cadmium selenide by XPS}

Rogelio Ospina ${ }^{1, a)}$, Sergio A. Rincón-Ortiz ${ }^{1}$ and Jhonatan Rodriguez-Pereira ${ }^{1,2}$

${ }^{1}$ Centro de Investigación Científica y Tecnológica en Materiales y Nanociencias (CMN),

Universidad Industrial de Santander, Piedecuesta, Santander, P.C. 681011, Colombia.

${ }^{2}$ Center of Materials and Nanotechnologies, Faculty of Chemical Technology, University of

Pardubice, Nam. Cs. Legii 565, 53002 Pardubice, Czech Republic.

(Received day Month year; accepted day Month year; published day Month year)

Cadmium selenide was analyzed using $\mathrm{x}$-ray photoelectron spectroscopy (XPS). The specimen is a powder purchased from Chemsavers. Sample was fixed to a stainless-steel sample holder with copper 3M $\mathrm{M}^{\mathrm{TM}}$ double-sided adhesive tape. Survey spectra, Cd 3d, Se 3d, O 1s, Cd 3p, Cd MNN, Se LMM, Se 3p, C 1s, and Cd 4d core level along with the valence band spectra were recorded. Results point out a stoichiometry of 0.95 on surface.

Keywords: CdSe; XPS; metal chalcogenide, semiconductor

\section{INTRODUCTION}

Due to their optical and electrical properties, metal chalcogenides are materials that can be used in different optoelectronic applications, as well as in electronic devices (transistors, diodes, etc.). Metal chalcogenides are chemical compounds between a transition metal and an anion chalcogen (S, Se, Te) (Refs 1-4). In this work, a sample of cadmium selenide (CdSe) was analyzed, which is a very popular material in this area because it has a band gap energy of $1.74 \mathrm{eV}$ (Ref. 4).

\section{SPECIMEN DESCRIPTION (ACCESSION \# 01637)}

Host Material: CdSe

CAS Registry \#: 1306-24-7

Host Material Characteristics: homogeneous; solid; polycrystalline; semiconductor; semiconductor; Powder

Chemical Name: Cadmium selenide

Source: Chemsavers

Host Composition: CdSe (99.999\%)

Form: Powder

Structure: CdSe

History \& Significance: CdSe powder was ground and fixed to a sample holder with copper $3 \mathrm{M}^{\mathrm{TM}}$ double-sided adhesive tape.

As Received Condition: As powder

Analyzed Region: same as host material

Ex Situ Preparation/Mounting: As received.

In Situ Preparation: None

Charge Control: Not applicable

Temp. During Analysis: $300 \mathrm{~K}$

Pressure During Analysis: $<1 \times 10^{-7} \mathrm{~Pa}$

Pre-analysis Beam Exposure: Not applicable s
Accession\#: 01637

Technique: XPS

Host Material: CdSe

Instrument: SPECS

PHOIBOS 150

Major Elements in

Spectra: Cd, Se

Minor Elements in Spectra: C, O

Published Spectra: 9

Spectra in Electronic Record: 9

Spectral Category:

comparison

\section{INSTRUMENT DESCRIPTION}

Manufacturer and Model: SPECS PHOIBOS 150 - SPECS Surface Nano Analysis GmbH

Analyzer Type: spherical sector

Detector: other

Number of Detector Elements: 25

INSTRUMENT PARAMETERS COMMON TO ALL SPECTRA

-Spectrometer

Analyzer Mode: constant pass energy

Throughput $\left(T=E^{N}\right): N=0$

Excitation Source Window: Mylar window, allows high Xray transmission: $88 \%$ for $\mathrm{Al} \mathrm{K} \alpha$.

Excitation Source: Al Ka monochromatic

Source Energy: $1486.6 \mathrm{eV}$

Source Strength: $200 \mathrm{~W}$

Source Beam Size: $2000 \mu \mathrm{m}$ x $2000 \mu \mathrm{m}$

Signal Mode: multichannel direct

-Geometry

Incident Angle: $55^{\circ}$

Source-to-Analyzer Angle: $55^{\circ}$

Emission Angle: $0^{\circ}$

Specimen Azimuthal Angle: Not applicable

Acceptance Angle from Analyzer Axis: $16^{\circ}$

Analyzer Angular Acceptance Width: $16^{\circ} \mathrm{x} 16^{\circ}$

-lon Gun

Manufacturer and Model: SPECS IQE 12/38

Energy: $5000 \mathrm{eV}$ 
Current: $70 \mathrm{~mA}$

Current Measurement Method: biased stage

Sputtering Species: $\mathrm{Ar}^{+}$

Spot Size (unrastered): $3000 \mu \mathrm{m}$ x $3000 \mu \mathrm{m}$

Raster Size: Not applicable $\mu \mathrm{m}$ x $\mu \mathrm{m}$

Incident Angle: $54^{\circ}$

Polar Angle: Not applicable

Azimuthal Angle: Not applicable

Comment: The specimen was analyzed as loaded. The ion gun was used only for cleaning the Ag reference foil.

\section{DATA ANALYSIS METHOD}

Energy Scale Correction: Binding energy scale was referenced to Cd 3d $d_{5 / 2}=405.0$ (Ref. 5).

Recommended Energy Scale Shift: $-0.11 \mathrm{eV}$

Peak Shape and Background Method: Peak position and width were determined from fitting the spectra using a mixed Gaussian- Lorentzian function after subtraction of a Shirley background using the CasaXPS Software.

Quantitation Method: Peak areas were obtained from fitting the spectra and relative sensitivity factors from the atomic photoionization cross section of each core level provided by SPECS Prodigy library.

\section{ACKNOWLEDGMENTS}

Authors thank to Laboratorio Central en Ciencia de Superficies (SurfLab-UIS) from Universidad Industrial de Santander for providing their facilities to perform the XPS measurements and to project 2321 of Vicerrectoría de Investigación y Extensión (VIE-UIS) from Universidad Industrial de Santander.

\section{REFERENCES}

1. D.H. Cho, W.J. Lee, S.W. Park, J.H. Wi, W.S. Han, J. Kim, M.H. Cho, D. Kim and Y.D. Chung, J. Mater. Chem. A. 2, 14593 (2014).

2. J.J. Wu and G.J. Lee, Advanced Nanomaterials for Green Energy (Elsevier, Netherlands, 2018), pp. 145, 167.

3. S.M. Kwon, J.K. Won, J.W. Jo, J. Kim, H.J. Kim, H.I. Kwon, J. Kim, S. Ahn, Y.H. Kim, M.J. Lee, H.I. Lee, T.J. Marks, M.G. Kim and S.K. Park, Sci. Adv. 4, 9104 (2018).

4. C.H. Rosmani, A.Z. Zainurul, M. Rusop and S. Abdullah. Adv. Mater. Res. 832, 557 (2014).

5. R. Islam and D.R. Rao, J. Electron Spectrosc. Relat. Phenom. 81, 69 (1996). 


\begin{tabular}{|c|c|c|c|c|c|c|c|}
\hline \multicolumn{8}{|c|}{ SPECTRAL FEATURES TABLE } \\
\hline $\begin{array}{c}\text { Spectrum } \\
\text { ID \# }\end{array}$ & $\begin{array}{l}\text { Element/ } \\
\text { Transition }\end{array}$ & $\begin{array}{c}\text { Peak } \\
\text { Energy } \\
(\mathrm{eV})\end{array}$ & $\begin{array}{c}\text { Peak } \\
\text { Width } \\
\text { FWHM } \\
(\mathrm{eV})\end{array}$ & $\begin{array}{l}\text { Peak Area } \\
(\mathrm{eV} \times \mathrm{cts} / \mathrm{s})\end{array}$ & $\begin{array}{l}\text { Sensitivity } \\
\text { Factor }\end{array}$ & $\begin{array}{c}\text { Concentration } \\
\text { (at. \%) }\end{array}$ & Peak Assignment \\
\hline 01637-02 & Cd 3d & $\ldots$ & $\ldots$ & $1.94 \times 10^{4}$ & 19.84 & 41.77 & $\ldots$ \\
\hline 01637-02 & $\mathrm{Cd} 3 \mathrm{~d}_{5 / 2}$ & 405.0 & 0.88 & $\ldots$ & $\ldots$ & $\ldots$ & CdSe \\
\hline 01637-02 & $\mathrm{Cd} 3 \mathrm{~d}_{3 / 2}$ & 411.7 & 0.86 & $\ldots$ & $\ldots$ & $\ldots$ & CdSe \\
\hline 01637-03 & Se 3d & $\ldots$ & $\ldots$ & $2.42 \times 10^{3}$ & 2.41 & 39.63 & $\ldots$ \\
\hline 01637-03 & Se $3 d_{5 / 2}$ & 53.8 & 0.92 & $\ldots$ & $\ldots$ & $\ldots$ & CdSe \\
\hline 01637-03 & Se $3 d_{3 / 2}$ & 54.6 & 0.87 & $\ldots$ & $\ldots$ & $\ldots$ & CdSe \\
\hline 01637-04 & $01 s$ & 531.5 & 2.50 & $9.45 \times 10^{2}$ & 2.77 & 18.60 & Contaminant \\
\hline 01637-05 & $\mathrm{Cd} 3 p_{3 / 2}$ & 617.9 & 3.49 & $8.92 \times 10^{3}$ & $\ldots$ & $\ldots$ & CdSe \\
\hline 01637-05 & $\mathrm{Cd} 3 \mathrm{p}_{1 / 2}$ & 651.8 & 3.40 & $4.46 \times 10^{3}$ & $\ldots$ & $\ldots$ & CdSe \\
\hline $01637-06^{a}$ & Cd MNN & 375.3 & 3.74 & $3.53 \times 10^{3}$ & $\cdots$ & $\cdots$ & $\mathrm{CdSe}$ \\
\hline $01637-06^{a}$ & Cd MNN & 381.9 & 1.88 & $1.50 \times 10^{3}$ & $\ldots$ & $\ldots$ & CdSe \\
\hline $01637-07^{a}$ & Se LMM & 1307.2 & 2.32 & $1.43 \times 10^{4}$ & $\cdots$ & $\cdots$ & CdSe \\
\hline 01637-07 & Se $3 p_{3 / 2}$ & 160.2 & 1.89 & $2.26 \times 10^{3}$ & $\ldots$ & $\ldots$ & CdSe \\
\hline 01637-07 & Se $3 p_{1 / 2}$ & 165.9 & 1.70 & $1.04 \times 10^{3}$ & $\ldots$ & $\ldots$ & CdSe \\
\hline $01637-07^{a}$ & Se LMM & 1348.2 & 2.14 & $7.61 \times 10^{3}$ & $\cdots$ & $\cdots$ & CdSe \\
\hline $01637-08^{a}$ & Se LMM + C 1s & 1202.1 & 1.54 & $4.29 \times 10^{3}$ & $\ldots$ & $\ldots$ & CdSe + Contaminant \\
\hline $01637-08^{a}$ & Se LMM & 1190.4 & 3.38 & $3.80 \times 10^{3}$ & $\cdots$ & $\cdots$ & CdSe \\
\hline 01637-09 & $\mathrm{Cd} 4 \mathrm{~d}$ & 10.9 & 1.42 & $3.54 \times 10^{3}$ & $\ldots$ & $\ldots$ & CdSe \\
\hline $01637-09^{b}$ & VBM & 1.7 & $\ldots$ & $\ldots$ & $\ldots$ & $\ldots$ & CdSe \\
\hline \multicolumn{8}{|c|}{$\begin{array}{l}\text { Peak energy reported as kinetic energy (KE) } \\
\text { b Valence band maximum (VBM) }\end{array}$} \\
\hline \multicolumn{8}{|c|}{ ANALYZER CALIBRATION TABLE } \\
\hline $\begin{array}{c}\text { Spectrum ID } \\
\#\end{array}$ & $\begin{array}{l}\text { Element/ } \\
\text { Transition }\end{array}$ & $\begin{array}{l}\text { Peak Energy } \\
(\mathrm{eV})\end{array}$ & $\begin{array}{l}\text { Peak Width } \\
\text { FWHM (eV) }\end{array}$ & \multicolumn{2}{|c|}{$\begin{array}{c}\text { Peak Area } \\
(\mathrm{eV} \times \mathrm{cts} / \mathrm{s})\end{array}$} & $\begin{array}{c}\text { Concentration } \\
\text { (at. \%) }\end{array}$ & $\begin{array}{c}\text { Peak } \\
\text { Assignment }\end{array}$ \\
\hline$\ldots$ & $\mathrm{Ag} \mathrm{3d_{5/2 }}$ & 368.3 & \multicolumn{2}{|c|}{0.50} & \multicolumn{2}{|c|}{$\ldots$} & $\ldots$ \\
\hline
\end{tabular}




\section{GUIDE TO FIGURES}

\begin{tabular}{|c|c|c|c|c|c|}
\hline $\begin{array}{l}\text { Spectrum } \\
\text { (Accession) \# }\end{array}$ & Spectral Region & Voltage Shift* & Multiplier & Baseline & Comment \# \\
\hline 01637-01 & Survey & - & 1 & 0 & 1 \\
\hline 01637-02 & Cd 3d & 0.11 & 1 & 0 & 1 \\
\hline $01637-03$ & Se 3d & 0.11 & 1 & 0 & 1 \\
\hline 01637-04 & O 1s & 0.11 & 1 & 0 & 1 \\
\hline 01637-05 & $\mathrm{Cd} 3 p$ & 0.11 & 1 & 0 & 1 \\
\hline $01637-06$ & Cd MNN & 0.11 & 1 & 0 & 1 \\
\hline 01637-07 & Se LMM, Se $3 p$ & 0.11 & 1 & 0 & 1 \\
\hline 01637-08 & Se LMM, C 1s & 0.11 & 1 & 0 & 1 \\
\hline 01637-09 & $\mathrm{Cd} 4 \mathrm{~d}, \mathrm{VB}$ & 0.11 & 1 & 0 & 1 \\
\hline
\end{tabular}

*Voltage shift of the archived (as-measured) spectrum relative to the printed figure. The figure reflects the recommended energy scale correction due to a calibration correction, sample charging, flood gun, or other phenomenon.

1. CdSe powder 


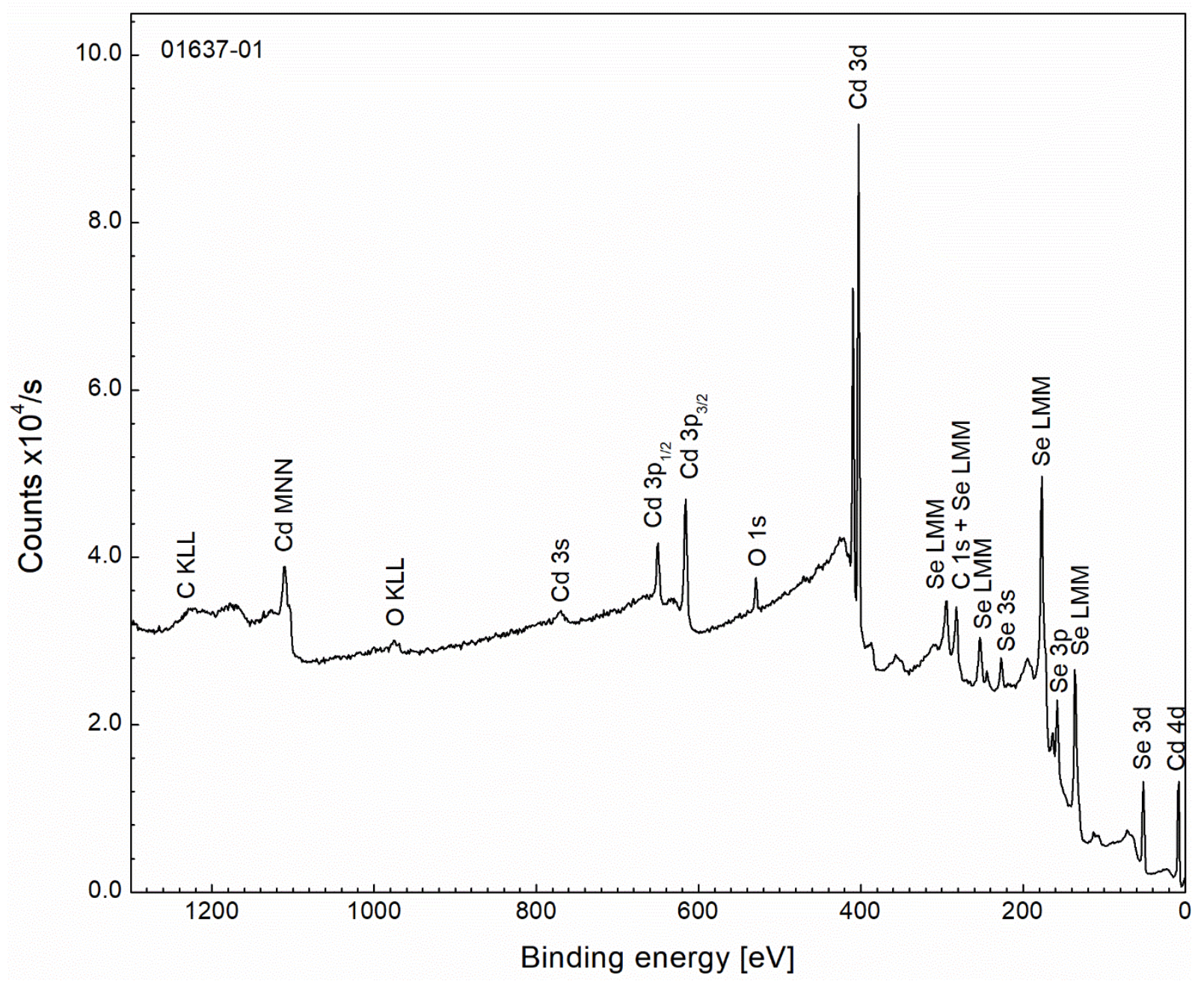

Publish in Surface Science Spectra: Yes $\otimes$ No $\square$

Accession \# 01637-01

Host Material

Technique

Spectral Region

Instrument

Excitation Source

Source Energy

Source Strength

Source Size

Analyzer Type

Incident Angle

Emission Angle

Analyzer Pass Energy

Analyzer Resolution

Total Signal Accumulation Time

Total Elapsed Time

Number of Scans

\section{CdSe \\ XPS \\ survey}

SPECS PHOIBOS 150

$\mathrm{Al} \mathrm{Ka} \mathrm{monochromatic}$

$$
\begin{gathered}
1486.6 \mathrm{eV} \\
200 \mathrm{~W} \\
2 \mathrm{~mm} \times 2 \mathrm{~mm}
\end{gathered}
$$

spherical sector analyzer

$55^{\circ}$
$0^{\circ}$
$100 \mathrm{eV}$
$0.5 \mathrm{eV}$
$122 \mathrm{~s}$
$260 \mathrm{~s}$
1
$5.28 \mathrm{eV}$




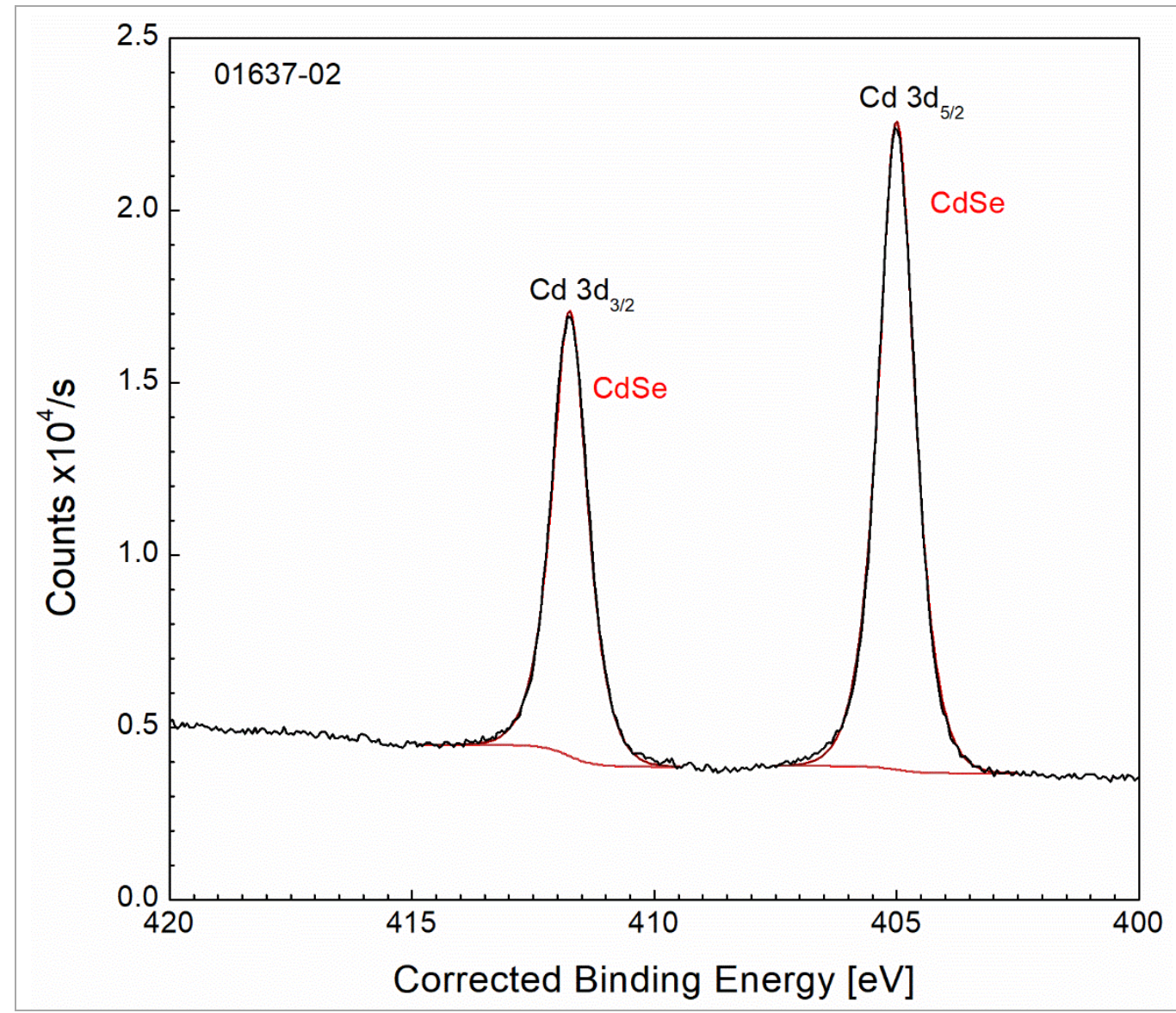

Publish in SSS: Yes $\triangle$ No $\square$

- Accession \#: 01637-02

- Host Material: CdSe

- Technique: XPS

- Spectral Region: Cd 3d Instrument: SPECS PHOIBOS 150

Excitation Source: Al Ka monochromatic

Source Energy: 1486.6 eV

Source Strength: $200 \mathrm{~W}$

Source Size: $2 \mathrm{~mm} \times 2 \mathrm{~mm}$

Analyzer Type: spherical sector Incident Angle: $55^{\circ}$

Emission Angle: 0

Analyzer Pass Energy 30 eV

Analyzer Resolution: $0.5 \mathrm{eV}$

Total Signal Accumulation Time: 432 $\mathrm{s}$

Total Elapsed Time: $720 \mathrm{~s}$

Number of Scans: 8

Effective Detector Width: $2.64 \mathrm{eV}$

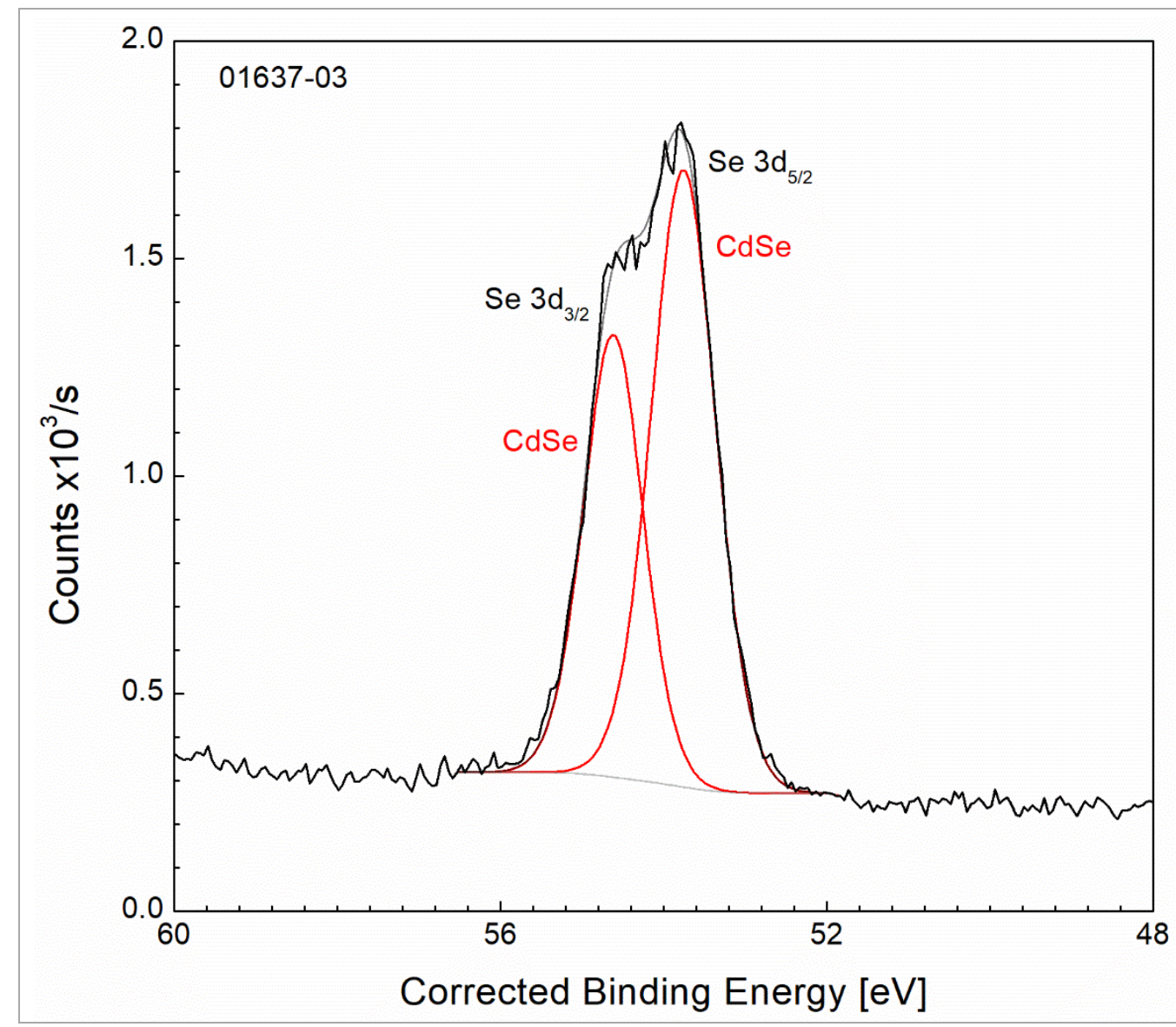

Publish in SSS: Yes $\triangle$ No $\square$

- Accession \#: 01637-03

- Host Material: CdSe

- Technique: XPS

- Spectral Region: Se 3d Instrument: SPECS PHOIBOS 150

Excitation Source: Al Ka monochromatic Source Energy: $1486.6 \mathrm{eV}$

Source Strength: $200 \mathrm{~W}$ Source Size: $2 \mathrm{~mm}$ x $2 \mathrm{~mm}$ Analyzer Type: spherical sector Incident Angle: $55^{\circ}$

Emission Angle: $0^{\circ}$ Analyzer Pass Energy $30 \mathrm{eV}$ Analyzer Resolution: $0.5 \mathrm{eV}$ Total Signal Accumulation Time: $225 \mathrm{~s}$ Total Elapsed Time: $420 \mathrm{~s}$ Number of Scans: 8 Effective Detector Width: $2.64 \mathrm{eV}$ 


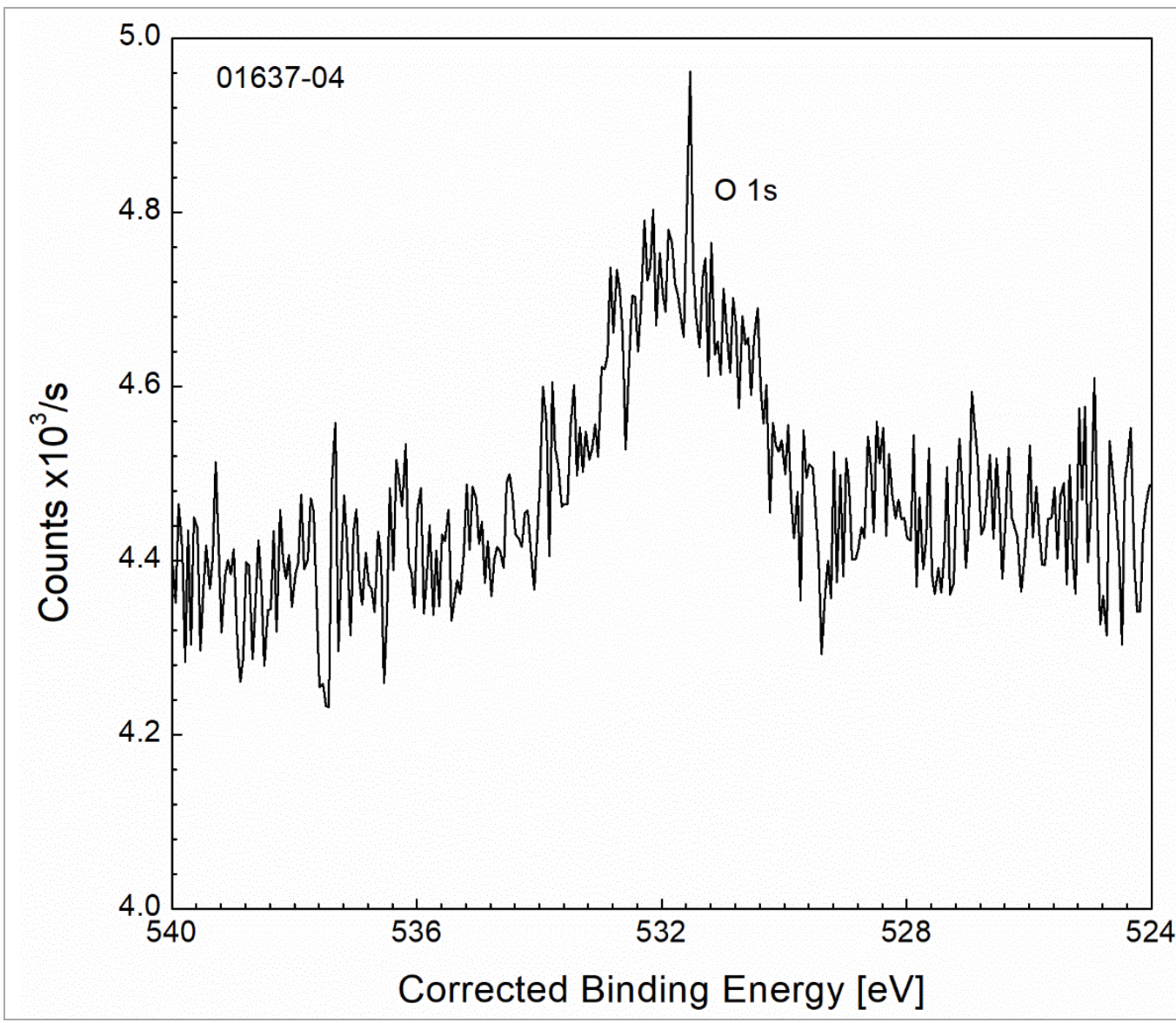

Publish in SSS: Yes $\otimes$ No $\square$

- Accession \#: 01637-04

- Host Material: CdSe

- Technique: XPS

- Spectral Region: $01 \mathrm{~s}$ Instrument: SPECS PHOIBOS 150

Excitation Source: Al Ka monochromatic

Source Energy: $1486.6 \mathrm{eV}$

Source Strength: $200 \mathrm{~W}$

Source Size: $2 \mathrm{~mm} \times 2 \mathrm{~mm}$

Analyzer Type: spherical sector

Incident Angle: $55^{\circ}$

Emission Angle: 0 •

Analyzer Pass Energy $30 \mathrm{eV}$

Analyzer Resolution: $0.5 \mathrm{eV}$

Total Signal Accumulation Time: 337 $\mathrm{s}$

Total Elapsed Time: 564 s

Number of Scans: 8

Effective Detector Width: $2.64 \mathrm{eV}$

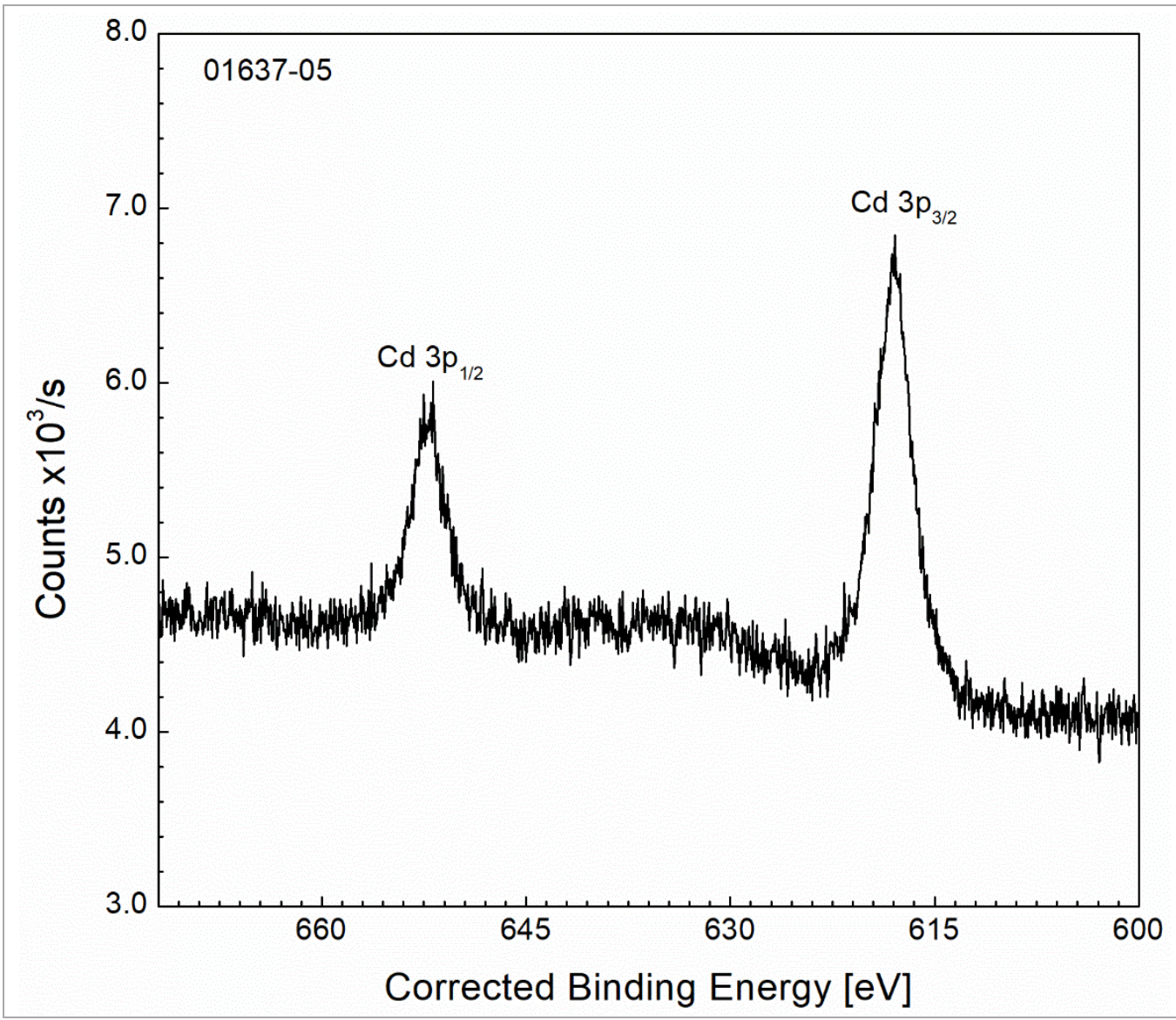

Publish in SSS: Yes $\triangle$ No $\square$

- Accession \#: 01637-05

- Host Material: CaSe

- Technique: XPS

- Spectral Region: Cd 3p Instrument: SPECS PHOIBOS 150

Excitation Source: Al Ka monochromatic

Source Energy: 1486.6 eV

Source Strength: $200 \mathrm{~W}$

Source Size: $2 \mathrm{~mm} \times 2 \mathrm{~mm}$

Analyzer Type: spherical sector Incident Angle: $55^{\circ}$

Emission Angle: $0^{\circ}$

Analyzer Pass Energy $30 \mathrm{eV}$ Analyzer Resolution: $0.5 \mathrm{eV}$

Total Signal Accumulation Time: 600 $\mathrm{s}$

Total Elapsed Time: $913 \mathrm{~s}$

Number of Scans: 4

Effective Detector Width: $2.64 \mathrm{eV}$ 


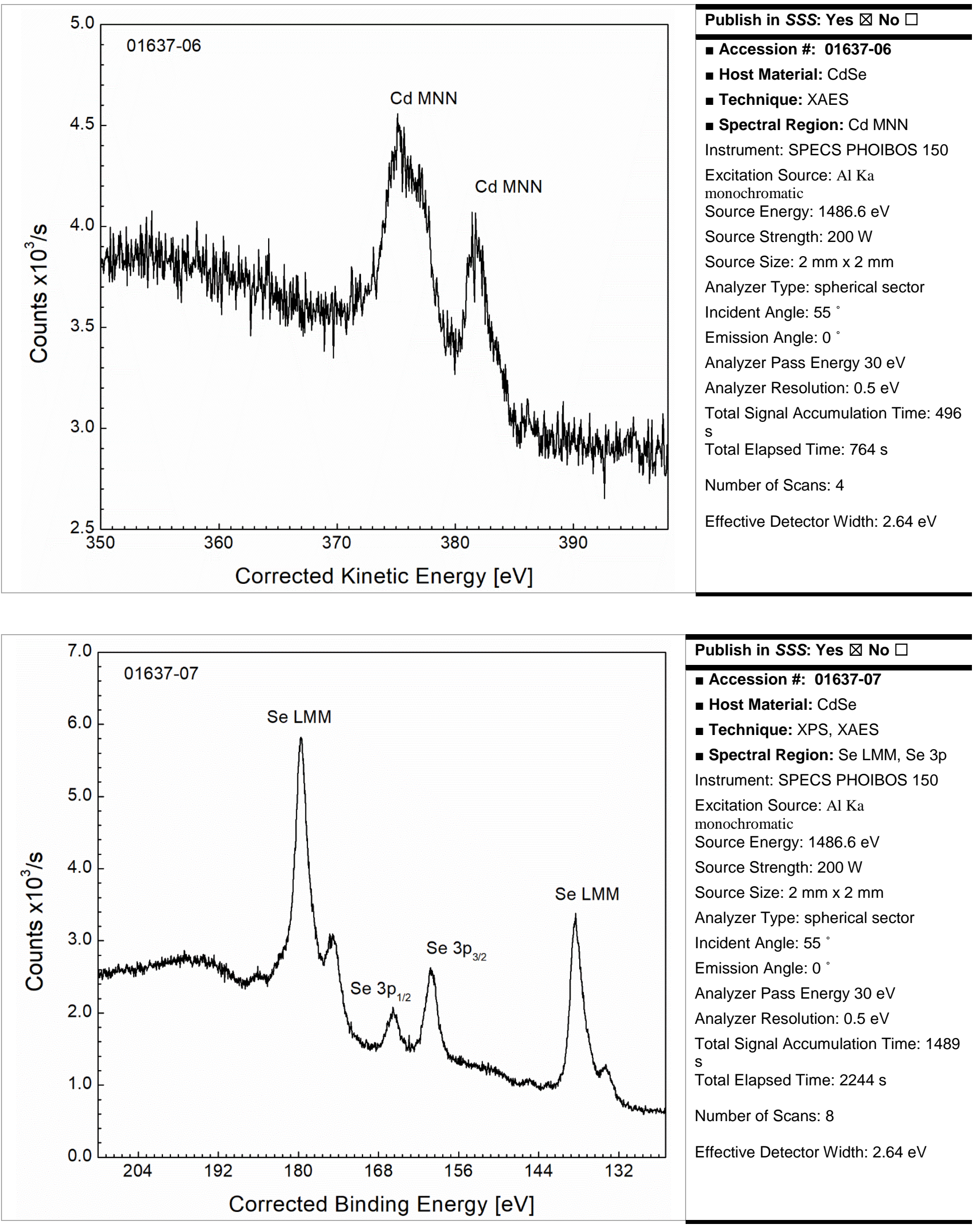



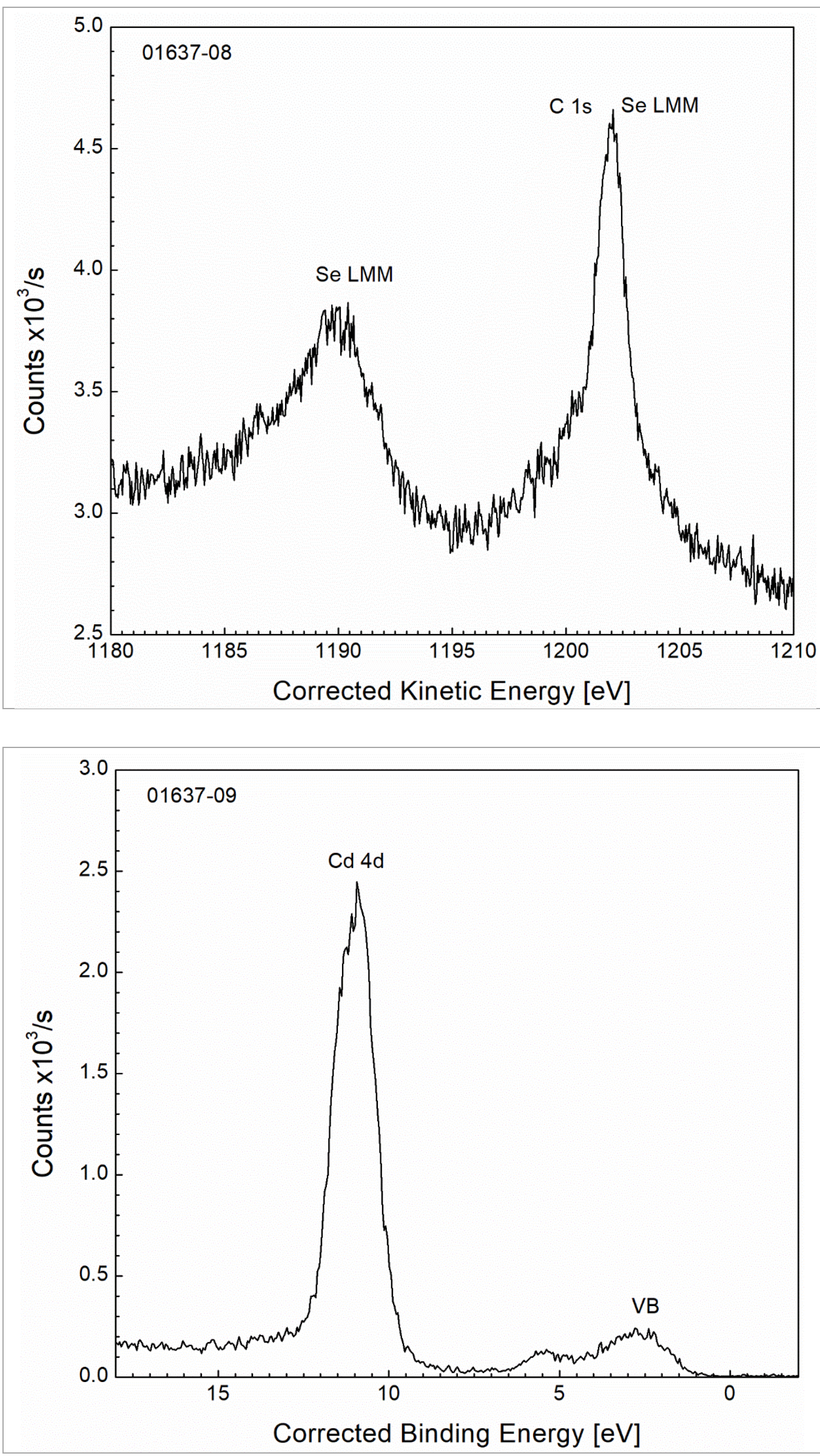

Publish in SSS: Yes $\otimes$ No $\square$

- Accession \#: 01637-08

- Host Material: CdSe

- Technique: XAES

- Spectral Region: C 1s, Se LMM Instrument: SPECS PHOIBOS 150

Excitation Source: Al Ka monochromatic

Source Energy: $1486.6 \mathrm{eV}$

Source Strength: $200 \mathrm{~W}$

Source Size: $2 \mathrm{~mm} \times 2 \mathrm{~mm}$

Analyzer Type: spherical sector Incident Angle: $55^{\circ}$

Emission Angle: $0^{\circ}$

Analyzer Pass Energy $30 \mathrm{eV}$

Analyzer Resolution: $0.5 \mathrm{eV}$

Total Signal Accumulation Time: 544 $\mathrm{s}$

Total Elapsed Time: 876 s

Number of Scans: 8

Effective Detector Width: $2.64 \mathrm{eV}$

Publish in SSS: Yes $\triangle$ No $\square$

- Accession \#: 01637-09

- Host Material: CdSe

- Technique: XPS

- Spectral Region: Cd 4d, VB Instrument: SPECS PHOIBOS 150

Excitation Source: Al Ka monochromatic

Source Energy: $1486.6 \mathrm{eV}$

Source Strength: $200 \mathrm{~W}$

Source Size: $2 \mathrm{~mm} \times 2 \mathrm{~mm}$

Analyzer Type: spherical sector Incident Angle: $55^{\circ}$

Emission Angle: $0^{\circ}$

Analyzer Pass Energy $30 \mathrm{eV}$

Analyzer Resolution: $0.5 \mathrm{eV}$

Total Signal Accumulation Time: $304 \mathrm{~s}$

Total Elapsed Time: $485 \mathrm{~s}$

Number of Scans: 4

Effective Detector Width: 2.64 eV 